\title{
The Correlation between Dietary Selenium Intake and Type 2 Diabetes: A Cross-Sectional Population-Based Study on North Chinese Adults
}

\author{
Sultan Mehmood Siddiqi $\mathbb{D}^{1},{ }^{1}$ Changhao Sun $\mathbb{D}^{1},{ }^{1}$ Xiaoyan $\mathrm{Wu},{ }^{1}$ Imranullah Shah, \\ and Anam Mehmood ${ }^{2}$ \\ ${ }^{1}$ Department of Nutrition and Food Hygiene, College of Public Health, Harbin Medical University, Harbin, \\ Heilongjiang Province 150081, China \\ ${ }^{2}$ Department of Clinical Psychology, College of Public Health, Harbin Medical University, Harbin, \\ Heilongjiang Province 150081, China
}

Correspondence should be addressed to Changhao Sun; sun2002changhao@126.com

Received 1 June 2019; Revised 2 November 2019; Accepted 19 November 2019; Published 22 January 2020

Academic Editor: Ken-ichi Aihara

Copyright (c) 2020 Sultan Mehmood Siddiqi et al. This is an open access article distributed under the Creative Commons Attribution License, which permits unrestricted use, distribution, and reproduction in any medium, provided the original work is properly cited.

\begin{abstract}
The relationship between selenium (Se) and type 2 diabetes (T2D) remains controversial. In previous animal and cell studies, Se was found to be insulin mimic and antidiabetic, whereas recent epidemiological and interventional trials have shown an unexpected association between high Se intake and increased risk of T2D. The present study aimed to investigate the significance of dietary Se and T2D in North Chinese adults. A large sample of the population was enrolled through cluster sampling in Northern China $(N=8824)$. Information on basic characteristics, anthropometric measures, and dietary Se intake was collected from each subject for analysis. Multivariable logistic regression was used to investigate the association between dietary Se and T2D through adjusted odds ratio (OR) and the corresponding 95\% confidence interval (CI). The average nutritional Se intake was $52.43 \mu \mathrm{g} / \mathrm{day}$, and the prevalence of T2D was $20.4 \%$ in the studied population. The OR for developing T2D was 1.66 (95\% CI: 1.38, 1.99 ; $P$ for linear trend <0.005), comparing the highest to the lowest quintile of energy-adjusted Se intake in multivariate logistic regression analysis. The mediation analysis discovered that glucose metabolism (indicated by FBG and HbA1c) mediated this association. In conclusion, our research adds further support to the role of high dietary Se in the incidence of T2D. The results also suggested that this association was mediated by glucose metabolism.
\end{abstract}

\section{Introduction}

Se is an essential micronutrient that plays structural and enzymatic roles in antioxidant defense systems, such as metabolism of thyroid hormone, red-ox homeostasis, reproduction, and immunity through Se-containing selenoproteins such as iodothyronine deiodinases, glutathione peroxidases (GPx), and thioredoxin reductases [1-3]. It has the potential to prevent the onset and progress of T2D, gestational disease, cardiovascular disease (CVD), [4] colorectal cancer, and prostate cancer by the action of its antioxidant selenoproteins and insulin mimic selenate [4-7]. Various prospective studies have claimed the reduction of diabetic risk with higher Se intake, suggesting a beneficial effect of Se supplementation $[7,8]$. Likewise, individuals with higher Se levels have a lower chance of T2D, as claimed in a prospective study on healthy men and women in the US population [7]. Furthermore, the higher intake of inorganic Se has a progressively negative association with T2D in various animal studies $[9,10]$.

However, some studies reported a nonsignificant association between Se and T2D [11, 12]. A cross-sectional survey on the Nord-Trondelag Health Survey found no evidence for the significant association of serum Se and T2D by analyzing the trace elements status in the blood of diabetic patients [13]. In contrast, other reports have 
significantly proved a positive association between Se and T2D $[14,15]$. The National Health and Nutrition Examination Survey of US (NHANES) reported an increased risk of T2D with the increase of serum Se in 1988-1994 [16] and further in 2003-2004 [14]. Our findings are also supported by a large sample ORDET cohort study of Northern Italian women that confirmed a significantly positive correlation between serum Se and T2D [17]. Likewise, a cross-sectional survey on the Asian population further confirmed that high dietary Se might be associated with the possible risk of T2D [18]. Besides, rats, mice, and pigs reported diabetic phenotypes with high Se diet (0.4 to $3.0 \mathrm{mg} / \mathrm{kg}$ ) [19]. Moreover, the findings of an observational study on a large sample of Chinese adults reported that Se supplementation might be positively connected with T2D [20].

Natural foods are enriched sources of Se such as cereals, seafood, organ meat, and crops. In China, Se intake tremendously varies among populations. For instance, Se is deficient in Europe but very high in Canada, Venezuela, the US, and Japan. Se level in the soil is affected by $\mathrm{pH}$, pesticides, rain, and evaporation. Dietary intake of Se considerably varies in the world due to inconsistent Se contents of plants, animal foods, and soil [21]. Se contents in a particular food depend upon the Se level in the soil where the food is grown, and the average recommended Se intake depends upon glutathione peroxidase potential activity [22]. Se intake in the United States is 60-220 $\mu \mathrm{g} / \mathrm{day}$, and the health benefits or metabolic toxicity of Se are not very clear at higher Se intake, more than the recommended dietary allowance (RDA) [21, 23]. In Europe, Se intake is comparatively lower than in the US but varies considerably between marginally adequate to adequate (30-90 $\mu \mathrm{g} /$ day) in Western and Central Europe and deficient to inadequate intake (7-30 $\mu \mathrm{g} /$ day) in Eastern European regions [21]. Few Chinese people studies have adjusted necessary dietary information and studied the association between dietary Se and T2D. Therefore, this study investigated the possible correlation between dietary Se and T2D in North Chinese adults.

\section{Materials and Methods}

2.1. Study Population. The Harbin Cohort Study on Diet, Nutrition, and Chronic Noncommunicable Diseases (HDNNCDS) is a population-based study launched at the nutrition and food hygiene department of Harbin Medical University by using similar survey methods as described in previous publications [24]. In this cross-sectional study, well-trained personals interviewed all the participants and collected demographic and health-related data by using a well-structured questionnaire during a medical examination. On that account, a total of 42 communities were indiscriminately selected from 3 strata in 7 urban administrative regions of Harbin, representing the baseline status of geographical atmosphere, economic situation, and social behavior. We decided the eligible participants (1) between 20 and 74 years, (2) residing in Harbin for not less than two years, and (3) without type 1 diabetes or cancer. Finally, 9,828 people participated in this study among 12,865 eligible participants, with a $76.4 \%$ participation rate. Reasons for not joining were unconcerned in the survey $(4.6 \%)$, restraints of time $(7.8 \%)$, poor study understanding $(10.1 \%)$, and no reason is given (1.1\%). 94 individuals were further excluded at baseline interview, who were found to be more or less than 74 and 20 years, respectively, and also those who had reported for extreme consumption of energy (men $>4,200$ or $<800 \mathrm{kcal} /$ day, women $>3,500$ or $<500 \mathrm{kcal} /$ day, $N=724$ ) and missing data for body mass index (BMI), education, waist circumference (WC), and Se intake $(N=186)$. After all these exclusions, the final study comprised of 8,824 subjects (3,187 men and 5,637 women). This study had been registered at chictr.org as ChiCTR-ECH-12002721.

2.2. Measurements. Relevant survey information was obtained in this cross-sectional study through structured questionnaires including (1) dietary habits, (2) physical examination and lifestyle, (3) and demographic measurements. A semiquantitative food frequency questionnaire model was used to evaluate the average daily dietary consumption rate over the past 12 months. This model included 14 different food groups, with 103 most popular and common food items consumed by Harbin people. We used Food Nutrition Calculator (V1.60, Chinese Center for Disease Control (CDC), Beijing, China) to estimate dietary Se intake ( $\mu \mathrm{g} /$ day) and total energy consumption (kcal/day) in different food items [25].

Well-structured lifestyle questionnaires were established to collect thorough information on disease history, family history of T2D (yes/no), age, education level (nonformal education, education in elementary school, middle or high school education, education in technical school or college, and university education), exercise (any kind of sports other than walking), cigarette smoking (smoked not less than 100 cigarettes over lifetime), alcohol consumption (consumed at least a bottle during last year), Se deficiency and supplementation history, occurrence of hyperlipidemia, coronary heart disease (CHD), and hypertension (systolic blood pressure $(\mathrm{SBP}) \geq 140 \mathrm{mmHg}$ or diastolic blood pressure $(\mathrm{DBP}) \geq 90 \mathrm{mmHg}$, and/or taking medications for hypertension).

Anthropometric measurements, including body weight, height, waist circumference (WC), and blood pressure (BP), were examined by well-trained personals. The average of three measurements was calculated with respective accuracy of $0.1 \mathrm{~kg}, 0.1 \mathrm{~cm}, 0.1 \mathrm{~cm}$, and $1 \mathrm{mmHg}$ by using standard conventional procedures. Body mass index $\left(\mathrm{kg} / \mathrm{m}^{2}\right)$ was measured by dividing the weight $(\mathrm{kg})$ with a square of the height $\left(\mathrm{m}^{2}\right)$. The fat mass (FM) analyzer analyzed the body fat by using the electric impedance method (OMRON HBF306, Omron Corporation, Dalian, China).

Homeostasis model assessment of insulin resistance (HOMA-IR) was calculated with the formula: fasting glucose $(\mathrm{mmol} / \mathrm{L}) \times$ fasting insulin $(\mathrm{mIU} / \mathrm{L}) / 22.5$, and HOMA-beta was calculated according to the method: $20 \times$ fasting insulin (mIU/L)/FPG (mmol/L)-3.5 [26]. Moreover, the haemoglobin A1c (HbA1c) assays were carried through using highperformance liquid chromatography (HPLC) on a Bio-Rad Variant VCS Hemoglobin Testing System (Shiga, Japan). 
The HbA1c intra-assay and interassay coefficients of variation were $0.7 \%$ at a value of $8.0 \%$ and $1.2 \%$ at a value of $5.8 \%$.

An OGTT was performed as per World Health Organization guidelines (WHO) [27]. Fasting and postprandial blood samples (blood samples taken after drinking water containing 75 grams of glucose) were collected with and kept at room temperature for 30 minutes. Samples were centrifuged for 5-10 minutes at a speed of $1,000 \mathrm{rpm} / \mathrm{min}$, and separated serum was collected for analysis. Incidence and prevalence of T2D were examined as follows: (1) by asking questions like "When the diagnosis confirmed the disease?" and "When were you diagnosed?" and "Did you control diabetes?" or (2) fasting blood glucose (FBG) $\geq 7.0 \mathrm{mmol} / \mathrm{L}$, and/or (3) $2 \mathrm{hr}$ glucose $\geq 11.1 \mathrm{mmol} / \mathrm{L}$ or (4) using diabetic medications.

2.3. Statistical Analysis. Quantitative and qualitative data were expressed in terms of mean \pm standard deviation (SD) and percentage, respectively. The study population was classified into energy-adjusted quintiles of dietary Se intake by using the residual method: $\leq 39.24,39.25-46.45,46.46-$ $52.57,52.58-60.68$, and $\geq 60.69 \mu \mathrm{g} /$ day. One-way variance analysis (uniformed data) and Kruskal-Wallis H-test (abnormal data) evaluated the divergences in the continuous data. Differences in qualitative data were measured by using the $\chi^{2}$ test. Logistic regression analysis assessed the ORs or the relative risk for developing T2D with $95 \%$ CI by comparing the quintiles of energy-adjusted Se with the lowest quintile serving as a reference. We used two core adjustment models: model 1 (partially adjusted model) adjusted for age, sex, BMI, waist; model 2 (fully customized model) additionally adjusted for smoking (past, current, never), alcohol intake, energy intake (nonalcohol), body fat, education, family history of diabetes, exercise, hypertension, and coronary heart disease. Logical regression analyzed the linear trend with Se as a median variable of each category. Subgroup analyses investigated the possible interaction between energy-adjusted Se and gender or BMI. Fully adjusted ORs with potential 95\% CIs were evaluated in the sex (male and female) and BMI subgroup ( $<25$ or $\geq 25)$.

The variations of insulin resistance in different intakes of dietary Se were analyzed across quintile of dietary Se. HOMA-IR and HOMA- $\beta$ were compared among groups using ANCOVA after controlling for age, sex, and BMI. Partial correlation between energy-adjusted dietary Se and markers of glucose metabolism (FINS, FBG, and HbA1c) was compared using ANCOVA after controlling for age, sex, and BMI.

Mediation analysis was performed to evaluate the role of HOMA-IR, HOMA- $\beta$, FBG, FINS (fasting insulin), and $\mathrm{HbAlc}$ as potential mediators of the association between dietary Se and T2D. And statistical significance for the mediation effect was carried out by formally testing for the average causal mediation effect, using bootstrapping techniques. The complete data analyses were carried out through SPSS 17.0 and R version 3.0.3 (http://www.r-project.org/), while $P<0.05$ was regarded to have statistical significance.

\section{Results}

The mean dietary Se intake of 8,824 participants was $52.43 \mu \mathrm{g} /$ day, indicating a relatively high baseline Se level. We observed that the participants with T2D were older and most likely the male with a higher level of BMI, body fat, protein, TG, cholesterol, energy intake, CHD, hypertension, obesity, and lower levels of carbohydrate than those in healthy individuals while nonsignificant differences were observed for fiber, cholesterol, and drinking (Table 1).

Wheat, livestock, fish, rice, and eggs are primary sources of dietary Se intake in the studied population (Table 2). Significant differences were assessed across quintiles of energy-adjusted Se intake in terms of BMI, sex, education, exercise, WC, consumption of alcohol, smoking status, and body fat. Meanwhile, age, energy intake, family history of diabetes, coronary heart disease, hypertension, hyperlipidemia, HOMA-IR, and HOMA- $\beta$ showed a nonsignificant association with energy-adjusted Se groups (Table 3).

The age, sex, BMI, waist adjusted OR for the highest quintile was 1.74 (95\% CI 1.46-2.07) when compared with the lowest quintile of energy-adjusted Se. It reveals a progressive increase in the risk of T2D $\left(P_{\text {trend }}<0.001\right)$. After multivariate adjustment for age, sex, BMI, waist, smoking (current, past, never), alcohol consumption, total energy intake, level of education, body fat, exercise, hypertension, family history of diabetes, and CHD, the adjusted OR for Q2, Q3, Q4, and Q5 were 1.34 (95\% CI 1.11-1.63), 1.20 (95\% CI 0.99-1.47), 1.39 (95\% CI 1.14-1.69), and 1.66 (95\% CI 1.38-1.99), respectively (Table 4). Seemingly, the adjusted OR is greater than 1, indicating a positive correlation between dietary Se intakes and T2D. While exercise is significantly associated with dietary selenium, it does not affect the association between dietary selenium and T2D. Table 4 explains the outcomes of subgroup analysis. It was observed that dietary Se intake was positively correlated with T2D in gender and BMI subgroups $\left(P_{\text {trend }}<0.05\right)$, indicating a risk factor for both genders.

The correlations between energy-adjusted dietary Se $(\mu \mathrm{g} /$ day) quintiles and HOMA-IR and HOMA- $\beta$ are presented in Table 5. Both HOMA-IR and HOMA- $\beta$ were nonsignificantly $(P>0.05)$ correlated with energy-adjusted Se quintiles after adjusting for age, sex, and BMI.

Table 6 explained the relationship of energy-adjusted dietary Se with HbA1c, FBG, and FINS (fasting insulin). It was observed that energy-adjusted dietary Se was directly correlated with $\mathrm{HbA1c}$ and FBG $(P<0.001)$. On the other hand, we did not find any significance for the level of FINS $(P>0.05)$.

In mediation assessment, we observed statistically significant mediation effects of FBG and HbAlc (0.5498095\% bias-corrected intervals: $0.29877,0.86451, P \leq 0.001$ for FBG, and $0.79426,95 \%$ bias-corrected intervals: 0.41340, 2.41389, $P=0.006$ for $\mathrm{HbA1c}$ ), suggesting that the association between dietary Se and T2D was potentially mediated by glucose metabolism while no statistically significant mediation effect was found for FINS, HOMA-IR, and HOMA- $\beta$ (Table 7). 
TABLE 1: Characteristics of participants according to diabetes status $(N=8,824)$.

\begin{tabular}{|c|c|c|c|}
\hline Selected characteristics & $\begin{array}{c}\text { No diabetes } \\
N=7,020\end{array}$ & $\begin{array}{c}\text { Diabetes } \\
N=1,804\end{array}$ & $P$ \\
\hline Age at recruitment (years) & $49.74 \pm 10.17$ & $54.90 \pm 9.32$ & $<0.001$ \\
\hline BMI $\left(\mathrm{kg} / \mathrm{m}^{2}\right)$ & $24.67 \pm 3.44$ & $26.14 \pm 3.60$ & $<0.001$ \\
\hline $\mathrm{WC}(\mathrm{cm})$ & $84.80 \pm 10.13$ & $89.88 \pm 9.85$ & $<0.001$ \\
\hline Education (\%) & & & $<0.001$ \\
\hline No formal education & 1.4 & 2.9 & \\
\hline Elementary school & 4.4 & 7.0 & \\
\hline Middle school & 21.8 & 28.4 & \\
\hline High school/secondary technical school & 32.6 & 31.0 & \\
\hline Technical school/college & 33.0 & 24.2 & \\
\hline Postgraduate degree or above & 0.8 & 0.3 & \\
\hline Male $(\%)$ & 34.0 & 44.3 & $<0.001$ \\
\hline Female $(\%)$ & 66.0 & 55.7 & $<0.001$ \\
\hline Exercised regularly (\%) & 45.3 & 53.5 & $<0.001$ \\
\hline Current smokers $(\%)$ & 54.7 & 46.5 & $<0.001$ \\
\hline Current drinker (\%) & 35.0 & 32.9 & $<0.001$ \\
\hline Family history of diabetes (\%) & 13.2 & 24.2 & $<0.001$ \\
\hline Hypertension (\%) & 76.634 .1 & 59.1 & $<0.001$ \\
\hline Coronary heart disease (\%) & 15.9 & 29.2 & $<0.001$ \\
\hline Hyperlipidemia (\%) & 20.2 & 38.9 & $<0.001$ \\
\hline Dietary Se intake ( $\mu \mathrm{g} /$ day $)$ & $51.81 \pm 22.97$ & $54.87 \pm 26.35$ & 0.001 \\
\hline HOMA-IR & $2.0 \pm 2.2$ & $4.14 \pm 4.87$ & $<0.001$ \\
\hline HOMA- $\beta$ & $89.85 \pm 96.29$ & $61.04 \pm 65.34$ & $<0.001$ \\
\hline Body fat & $30.28 \pm 5.69$ & $31.29 \pm 6.10$ & $<0.001$ \\
\hline Energy & $2239.25 \pm 655.93$ & $2216.02 \pm 688.26$ & 0.184 \\
\hline
\end{tabular}

BMI: body mass index; WC: waist circumference; Se: selenium; HOMA-IR: homeostasis model assessment of insulin resistance; HOMA- $\beta$ : homeostasis model assessment beta. Data are mean \pm standard deviation, unless otherwise indicated. $P$ values are for test of difference between different diabetic statuses.

TABle 2: Food sources of selenium consumed by North Chinese people.

\begin{tabular}{lc}
\hline Food & $\%$ \\
\hline Rice & 13.38 \\
Wheat & 22.36 \\
Potatoes & 2.09 \\
Beans & 0.71 \\
Vegetables & 5.19 \\
Fruits & 1.51 \\
Livestock and poultry & 20.99 \\
Milk & 2.20 \\
Eggs & 11.30 \\
Fish & 18.86 \\
Snacks & 0.70 \\
Beverages & 0.35 \\
Ice cream & 0.36 \\
\hline
\end{tabular}

\section{Discussion}

We observed a significant linear association between dietary Se intake and an increased risk of T2D. This observational study was carried out in a large sample of the population who belongs to Harbin city in Heilongjiang province of Northern China, comprising 8,824 adults between the ages of 20 and 74 years. Several studies found that healthy people have higher Se levels than people with T2D $[6,7,28,29]$. Various interventional and epidemiological studies also drew conflicting conclusions on the association between T2D and Se level in different biomarkers [7, 23, 30, 31]. In a double-blinded, randomized, placebo-controlled model, the subjected population was supplemented with $200 \mu \mathrm{g}$ of Se intake for consecutive six weeks starting from 24-28 weeks of gestation. Consequently, a protective effect was demonstrated on levels of high sensitivity C-reactive protein, glucose metabolism, and oxidative stress biomarkers [5]. The finding of Suresh et al. [32] further explained these observations by indicating that decreased level of serum Se may lead to tissue destruction and inflammation in patients with T2D. An animal trial investigated that high Se exposure may induce reverse regulation of reactive oxygen species, demonstrating a hepatic insulin resistance [33].

In contrast, recent findings from cross-sectional studies in China [18], prospective studies in Europe [17], and observational and clinical outcomes from the US [16] indicate that Se supplementation or high Se level might be the possible risk of T2D. Various studies observed the association between Se intake (supplementary or dietary) and T2D with inconsistent conclusions. The previous three crosssectional studies concluded mixed results [14, 34, 35]. Only one study supported our findings and investigated the possible risk of high dietary Se in the prevalence of T2D in Chinese people [35]. Moreover, a randomized, placebocontrolled study explores the adverse impact of Se supplementation on glucose homeostasis in diabetic patients [36]. The third National Health and Nutrition Examination Survey (NHANES III) [16] and NHANES 2003-2004 [14] estimated a risk factor of T2D with high Se level. A previous meta-analysis [37] further confirmed this association, although it fails to reach statistical significance, with pooled relative risk 1.06 (95\% CI 0.97-1.15). Contrarily, several 
TAвLE 3: Characteristics of participants according to energy-adjusted quintiles of dietary selenium intake $(N=8,824)$.

\begin{tabular}{|c|c|c|c|c|c|c|}
\hline \multirow[b]{2}{*}{ Selected characteristics } & \multicolumn{5}{|c|}{ Quintiles of dietary Se intake ( $\mu \mathrm{g} /$ day) } & \multirow[b]{2}{*}{$P_{\text {trend }}$} \\
\hline & $\begin{array}{c}\mathrm{Q} 1 \\
N=1,764\end{array}$ & $\begin{array}{c}\mathrm{Q} 2 \\
N=1,766\end{array}$ & $\begin{array}{c}\mathrm{Q} 3 \\
N=1,765\end{array}$ & $\begin{array}{c}\mathrm{Q} 4 \\
N=1,764\end{array}$ & $\begin{array}{c}\text { Q5 } \\
N=1,765\end{array}$ & \\
\hline Age at recruitment (years) & 50.3 & 50.99 & 50.69 & 51.14 & 50.88 & 0.126 \\
\hline BMI $\left(\mathrm{kg} / \mathrm{m}^{2}\right)$ & 25.25 & 24.86 & 24.83 & 24.87 & 25.03 & 0.002 \\
\hline $\mathrm{WC}(\mathrm{cm})$ & 86.84 & 85.51 & 85.03 & 85.49 & 86.31 & $<0.001$ \\
\hline Education (\%) & & & & & & $<0.001$ \\
\hline No formal education & 2.0 & 1.5 & 1.5 & 1.6 & 1.9 & \\
\hline Elementary school & 7.4 & 5.0 & 4.8 & 4.0 & 3.2 & \\
\hline Middle school & 25.7 & 22.8 & 23.3 & 21.7 & 22.3 & \\
\hline High school/secondary technical school & 30.9 & 33.6 & 31.4 & 32.9 & 32.6 & \\
\hline Technical school/college & 28.2 & 29.9 & 32.0 & 33.0 & 33.2 & \\
\hline Male $(\%)$ & 40.5 & 31.3 & 30.3 & 34.9 & 43.6 & $<0.001$ \\
\hline Female (\%) & 59.5 & 68.7 & 69.7 & 65.1 & 56.4 & $<0.001$ \\
\hline Exercised regularly (\%) & 44.2 & 47.0 & 47.1 & 49.7 & 46.8 & 0.032 \\
\hline Current smokers (\%) & 20.5 & 15.3 & 14.8 & 15.9 & 21.2 & $<0.001$ \\
\hline Current drinker (\%) & 35.0 & 32.0 & 32.8 & 33.0 & 40.1 & 0.046 \\
\hline Family history of diabetes (\%) & 14.8 & 16.5 & 15.6 & 15.8 & 14.7 & 0.575 \\
\hline Hypertension (\%) & 41.6 & 37.6 & 38.4 & 37.9 & 40.4 & 0.065 \\
\hline Coronary heart disease (\%) & 19.3 & 17.8 & 20.1 & 18.2 & 17.8 & 0.288 \\
\hline Hyperlipemia (\%) & 25.1 & 24.3 & 24.3 & 23.5 & 22.7 & 0.528 \\
\hline HOMA-IR & 2.46 & 2.26 & 2.61 & 2.38 & 2.60 & 0.051 \\
\hline HOMA- $\beta$ & 90.59 & 83.70 & 84.19 & 82.74 & 79.96 & 0.132 \\
\hline Body fat & 30.40 & 30.77 & 30.67 & 30.59 & 29.99 & $<0.001$ \\
\hline Energy & 2633.57 & 2170.74 & 2026 & 1987.91 & 2354.39 & 0.184 \\
\hline
\end{tabular}

BMI: body mass index; WC: waist circumference; Se: selenium; HOMA-IR: homeostasis model assessment of insulin resistance; HOMA- $\beta$ : homeostasis model assessment beta. Data are mean \pm standard deviation, unless otherwise indicated. $P$ values are for test of difference across all quartiles of selenium intake.

TABle 4: Odds ratios $(95 \% \mathrm{CI})$ of incident type 2 diabetes by quintiles of dietary selenium intake in the total population, gender, and BMI subgroups.

\begin{tabular}{|c|c|c|c|c|c|c|}
\hline \multirow{2}{*}{ Selected characteristics } & \multicolumn{5}{|c|}{ Quintiles of dietary Se intake ( $\mu \mathrm{g} /$ day) } & \multirow{2}{*}{$P_{\text {trend }}$} \\
\hline & Q1 & Q2 & Q3 & Q4 & Q5 & \\
\hline Range of Se intake & $\leq 39.24$ & $39.25-46.45$ & $46.46-52.57$ & $52.58-60.68$ & $>60.69$ & \\
\hline No. of cases & 285 & 369 & 338 & 378 & 434 & \\
\hline \multicolumn{7}{|l|}{ Total population } \\
\hline Reduced model OR & 1.00 & $1.48(1.23-1.76)$ & $1.35(1.13-1.62)$ & $1.51(1.26-1.80)$ & $1.74(1.46-2.07)$ & $<0.001$ \\
\hline${ }^{*}$ Multivariable adjusted OR & 1.00 & $1.34(1.11-1.63)$ & $1.20(0.99-1.47)$ & $1.39(1.14-1.69)$ & $1.66(1.38-1.99)$ & $<0.001$ \\
\hline \multicolumn{7}{|l|}{ Sex subgroup } \\
\hline Male & 1.00 & $1.62(1.23-2.14)$ & $1.21(0.91-1.62)$ & $1.29(0.98-1.71)$ & $1.45(1.12-1.89)$ & 0.024 \\
\hline Female & 1.00 & $1.33(1.03-1.72)$ & $1.39(1.08-1.80)$ & $1.73(1.34-2.23)$ & $2.14(1.65-2.77)$ & $<0.001$ \\
\hline \multicolumn{7}{|l|}{ BMI subgroup } \\
\hline $\mathrm{BMI}<25$ & 1.00 & $1.57(1.16-2.13)$ & $1.42(1.04-1.93)$ & $1.75(1.30-2.36)$ & $2.12(1.57-2.85)$ & $<0.001$ \\
\hline $\mathrm{BMI} \geq 25$ & 1.00 & $1.32(1.06-1.62)$ & $1.19(0.94-1.55)$ & $1.38(1.10-1.71)$ & $1.57(1.24-1.98)$ & $<0.001$ \\
\hline
\end{tabular}

Se: selenium; OR: odds ratio. Reduced model: age, sex, BMI, waist. ${ }^{*}$ Multivariable adjusted: age, sex, BMI, WC, education smoking (past, current, never), alcohol intake, energy intake (nonalcohol), body fat, education, family history of diabetes, exercise, hypertension, and coronary heart disease.

TABLE 5: Insulin resistance according to dietary Se intake.

\begin{tabular}{|c|c|c|c|c|c|}
\hline & Q1 & Q2 & Q3 & Q4 & Q5 \\
\hline Number & $N=1764$ & $N=1766$ & $N=1765$ & $N=1764$ & $N=1765$ \\
\hline Se $(\mu \mathrm{g} /$ day $)$ & $\begin{array}{c}\leq 39.24 \\
r(p)\end{array}$ & $\begin{array}{c}39.25-46.45 \\
r(p)\end{array}$ & $\begin{array}{c}46.46-52.57 \\
r(p)\end{array}$ & $\begin{array}{c}52.58-60.68 \\
r(p)\end{array}$ & $\begin{array}{c}>60.69 \\
r(p)\end{array}$ \\
\hline HOMA-IR & $-0.009885(0.755)$ & $-0.021618(0.496)$ & $0.012121(0.698)$ & $0.030804(0.330)$ & $-0.010850(0.743)$ \\
\hline НОМА- $\beta$ & $0.022180(0.442)$ & $0.030418(0.339)$ & $-0.006698(0.830)$ & $0.013095(0.678)$ & $0.016879(0.611)$ \\
\hline
\end{tabular}

Partial correlations between energy-adjusted dietary Se ( $\mu$ g/day) quintiles and insulin resistance after controlling for age and BMI. HOMA-IR: homeostasis model assessment of insulin resistance; HOMA- $\beta$ : homeostasis model assessment of $\beta$ cell function, $r$ : partial correlation coefficient. 
TABLE 6: Correlations between dietary Se intake and glucose metabolism.

\begin{tabular}{lcr}
\hline & $r 0(p)$ & $r 1(p)$ \\
\hline FINS (pmol/L) & $-0.000105(0.994)$ & $0.057944(0.978)$ \\
FBG (mmol/L) & $0.046490(0.001)$ & $0.053343(0.001)$ \\
HbAlc & $0.057944(0.001)$ & $0.053372(0.001)$ \\
\hline
\end{tabular}

Partial correlations between dietary Se intake ( $\mu \mathrm{g} / \mathrm{day})$ and insulin resistance after controlling for age, sex, BMI. FINS: fasting insulin; FBG: fasting blood glucose; HbAlc: haemoglobin A1c; $r 0$ : correlation coefficient; $r 1$ : partial correlation coefficient.

TABle 7: Causal associations between dietary Se and T2D in HDNNCDS.

\begin{tabular}{lccc}
\hline Potential mediator & The total effects $(95 \% \mathrm{CI})$ & Proportion via mediation $(95 \% \mathrm{CI})$ & $\begin{array}{c}\text { Prop. mediated } \\
P \text { value }\end{array}$ \\
\hline FBG & $0.00086(0.00046,0.00127)$ & $0.54980(0.29877,0.86451)$ & $<0.001$ \\
HbA1c & $0.00063(0.00016,0.00116)$ & $0.79426(0.41340,2.41389)$ & 0.006 \\
FINS & $0.00077(0.00027,0.00132)$ & $-0.00020(-0.07005,0.06494)$ & 0.986 \\
HOMA-IR & $0.00076(0.00024,0.00119)$ & $0.04835(-0.08660,0.21473)$ & 0.328 \\
HOMA- $\beta$ & $0.00077(0.00029,0.00124)$ & $0.04563(-0.03614,0.17352)$ & 0.202 \\
\hline
\end{tabular}

FBG: fasting blood glucose; HbA1c: haemoglobin A1c; FINS: fasting insulin; HOMA-IR: homeostasis model assessment of insulin resistance; HOMA- $\beta$ : homeostasis model assessment of $\beta$ cell function.

studies assessed a nonsignificant association between Se and T2D $[38,39]$. A PRECISE pilot trial in the UK evaluated that intervention with 100,200 , or $300 \mu \mathrm{g} /$ day of $\mathrm{Se}$ for six months did not show any diabetic effects in participants with relatively low Se levels [39]. Nevertheless, the outcomes of Se and Vitamin E in Cancer Trial also (SELECT) confirmed a nonsignificant association between Se supplementation and T2D [16].

Various cross-sectional studies [14, 40, 41], longitudinal analyses, and controlled trials [35, 42, 43] support the findings in the present study, suggesting a statistically significant positive correlation between dietary Se and T2D. Moreover, a cross-sectional survey on Asian population further explored a decisive significance for the prevalence of T2D with high dietary Se intake [18].

The dietary Se is nonsignificantly associated with HOMA- $\beta$ and HOMA-IR in the present study. The association between Se nutritional status and insulin resistance is very complicated and intriguing. A negative association was found between Se and HOMA-IR in Korean [44] and Turkish populations [45]. In contrast, serum Se and HOMAIR reported a positive correlation in obese Egyptian children [46] and elderly Polish men with MS [47]. However, a nonsignificant association was found between serum Se and insulin resistance in a Swedish study [48]. The present study revealed that the relationship between dietary Se and the prevalence of $\mathrm{T} 2 \mathrm{D}$ might be mediated by glucose metabolism instead of insulin resistance.

There is limited mechanistic evidence that may clarify the relationship between increased Se exposure and high risk of T2D; therefore, at present, any such discussion is highly speculative. Se has high interindividual variability and narrow therapeutic range in terms of metabolic sensitivity $[49,50]$. Se forms such as selenate and selenite may impair the insulin signaling in rats and stimulate the catabolic activity in muscles with the increase of glycogen consumption and glycolysis rate [51]. High dietary Se may stimulate overexpression of glutathione peroxidase-1 (GPX-
1) and other antioxidant selenoproteins, promoting insulin resistance and obesity [52-54] or may induce the release of glucagon resulting in hyperglycemia [55]. Likewise, in humans, a group of nondiabetic pregnant women showed a strong positive association between GPx activity and insulin resistance [43]. From a mechanistic explanation, Se intakes higher than the recommended level for optimal antioxidant activity of selenoproteins, such as glutathione peroxidases $(55 \mu \mathrm{g} /$ day, serum or plasma concentrations $70-90 \mu \mathrm{g} / \mathrm{L})$ $[22,56]$, will lead to the nonspecific binding of selenomethionine substituting methionine in albumin and other proteins [3]. Other Se-dependent proteins, including selenoprotein $\mathrm{P}[57,58]$, methionine-R-sulfoxide reductase B1 [59], thioredoxin reductase 3 [59], and selenoprotein S [60], may also be involved in glucose metabolism or T2D. The metabolic pathways involved in this extra Se pool have not been fully understood and may be responsible for some of the adverse effects of high Se exposure on glucose metabolism.

In this study, we observed that Se has a significant positive association with the level of HbA1c and FBG. On the other hand, we did not find any changes at the level of FINS. Our results are supported by a recent population-based study [61], suggesting that subjects in higher quintiles of serum Se had an increased risk of T2D (higher levels of FBG and $\mathrm{HbA1c}$ ) compared with those in the first quartile. However, FBG reflects the glycemic status and HbA1c is regarded as a more reliable marker since it indicates longterm changes in the glycemic control. Thus, the impact of Se on HbA1c levels observed in this study confirms that Se is involved in the metabolism of glucose. Another study investigated the association between high serum Se levels and impaired fasting glucose and elevated fasting serum glucose [20]. Several studies reported that patients with T2D had higher serum Se concentration compared with healthy subjects, and serum Se levels had positively correlated with plasma glucose in T2D [14, 16, 42]. The link between Se and glucose seems to be very complicated and is supposed to be 
reflected by a nonlinear U-shaped dose-response relationship [62].

Moreover, a comprehensive study of the relationship between Se and T2D [63] concluded that this complicated relationship could be interpreted as a potential hazard of optimal activity of some or all Se-containing proteins at different physiological ranges. Current and previous studies have been conducted in participants with an average dietary Se intake close to the recommended nutritional intake, but this association may differ among people with deficient nutritional Se intake. Results of previous investigations intimated a U-shaped correlation between dietary Se and T2D. Thus, an imbalance in the physiological range of dietary Se might be a risk factor for the prevalence of T2D. A recent study on US adults explored the risk of supplementary Se, but this statistical significance was only confirmed in males in NHANES III [14].

The main strength of this survey is that it is the first and most extensive study to date to study the correlation between dietary Se intake and T2D of more than 8,800 people in Northern China. Most of the previous studies confirm this association in Europe or the US. Notably, it is utile to conduct this study on the north Chinese population because results may be affected due to differences in culture, geography, and dietary habits. Most strikingly, it is the first cross-sectional design to inquire about the link between dietary Se intake and T2D with large-scale north Chinese population and consistently supported by previous outcomes.

However, this study also has some limitations. First, a causality of relationship is not well defined in this crosssectional evaluation, so there is a need to verify our outcomes through additional prospective designs. Second, it was hard to measure the Se level in plasma or serum due to limited resources. However, in the environment with substantial changes in Se content of food and high frequency of using mineral and vitamin supplements, the use of questionnaire data to estimate Se intake has attracted attention. First, HDNNCDS developed a detailed database to analyze the Se content of local foods with ad hoc measurements. Secondly, the range of dietary Se intake in this study is consistent with the previous measurement results of $\mathrm{Se}$ status of the Chinese population-based on biomarkers [64]. It is easier to estimate Se intake through questionnaire survey data, while previous studies have analyzed the Se contents and Se status in various foods from all over China with minimal variability $[18,65]$. Finally, [24] no user of Se supplements was detected in our survey. In our settings, the evaluation of Se exposure by assessing dietary intake may even be more advantageous than Se biomarkers, which may be affected by Se own chemical species [56], smoking and other lifestyle variables [66], other nutrients (such as methionine) [67], and drug use [66]. Studying the relationship between dietary Se, serum Se, and T2D could help us to understand this topic more comprehensively. Population bias is another potential limitation of this analysis. Participants undergoing medical health surveys may not be able to confirm the representation of the overall population. However, the relationship between dietary Se and T2D was successfully measured by using large sample size, multivariate logistic regression model, and a comprehensive adjustment of confounding factors. The prevalence of T2D in the targeted population was $20.4 \%$. That is much higher than the computed value of the representative sample of Chinese adults in 2010, concluding a gradual increase in the prevalence of $\mathrm{T} 2 \mathrm{D}$.

\section{Conclusion}

The present study adds to the evidence of a positive correlation between dietary Se intake and incidence of T2D in the target population. The epidemiological evidence for a diabetogenic effect of Se suggests that Se rich foods should be consumed carefully, considering individual dietary requirements. Further work aimed to investigate the potential links between dietary Se and exposure of T2D needs to be further assessed on a priority basis.

\section{Data Availability}

All research data are available and will be provided on request to the corresponding author.

\section{Conflicts of Interest}

The authors declare that they have no conflicts of interest.

\section{Authors' Contributions}

Changhao Sun and Xiaoyan Wu conceived the idea. Sultan Mehmood Siddiqi designed the study and wrote the original manuscript. Imranullah Shah and Anam Mehmood were responsible for retrieving and analyzing the data. Anam Mehmood approved the version to be published. All authors critically assessed and reviewed the paper.

\section{Acknowledgments}

The authors are indebted to the participants of HDNNCDS for their continued cooperation and participation. The National Key R\&D Program of China (2017YFC1307401 to Changhao Sun) provided financial support for this study.

\section{References}

[1] M. P. Rayman, "The importance of selenium to human health," The Lancet, vol. 356, no. 9225, pp. 233-241, 2000.

[2] F. F. Chu, J. H. Doroshow, and R. S. Esworthy, "Expression, characterization, and tissue distribution of a new cellular selenium-dependent glutathione peroxidase, GSHPx-GI," The Journal of Biological Chemistry, vol. 268, no. 4, pp. 2571-2576, 1993.

[3] R. F. Burk, "Selenium, an antioxidant nutrient," Nutrition in Clinical Care, vol. 5, no. 2, pp. 75-79, 2002.

[4] S. Czernichow, A. Couthouis, S. Bertrais et al., "Antioxidant supplementation does not affect fasting plasma glucose in the supplementation with antioxidant vitamins and minerals (SU.VI.MAX) study in France: association with dietary intake and plasma concentrations," The American Journal of Clinical Nutrition, vol. 84, no. 2, pp. 395-399, 2006. 
[5] Z. Asemi, M. Jamilian, E. Mesdaghinia, and A. Esmaillzadeh, "Effects of selenium supplementation on glucose homeostasis, inflammation, and oxidative stress in gestational diabetes: randomized, double-blind, placebo-controlled trial," Nutrition, vol. 31, no. 10, pp. 1235-1242, 2015.

[6] E. A. Klein, I. M. Thompson, C. M. Tangen et al., "Vitamin E and the risk of prostate cancer: the selenium and vitamin $\mathrm{E}$ cancer prevention trial (SELECT)," JAMA, vol. 306, no. 14, pp. 1549-1556, 2011.

[7] S. Rajpathak, E. Rimm, J. S. Morris, and F. Hu, "Toenail selenium and cardiovascular disease in men with diabetes," Journal of the American College of Nutrition, vol. 24, no. 4, pp. 250-256, 2005.

[8] T. N. Akbaraly, J. Arnaud, M. P. Rayman et al., "Plasma selenium and risk of dysglycemia in an elderly French population: results from the prospective epidemiology of vascular ageing study," Nutrition \& Metabolism, vol. 7, no. 1, p. 21, 2010.

[9] A. S. Mueller, J. Pallauf, and J. Rafael, "The chemical form of selenium affects insulinomimetic properties of the trace element: investigations in type II diabetic dbdb mice," The Journal of Nutritional Biochemistry, vol. 14, no. 11, pp. 637647, 2003.

[10] R. Shimizu, H. Ueno, T. Okuno, F. Sakazaki, and K. Nakamuro, "Effect of sodium selenite supplementation on glucose intolerance and pancreatic oxidative stress in type 2 diabetic mice under different selenium status," Journal of Health Science, vol. 55, no. 2, pp. 271-280, 2009.

[11] H. Misu, K. Ishikura, S. Kurita et al., "Inverse correlation between serum levels of selenoprotein $\mathrm{P}$ and adiponectin in patients with type 2 diabetes," PLoS One, vol. 7, no. 4, Article ID e34952, 2012.

[12] M. Navarro-Alarcón, H. L.-G. de la Serrana, V. Pérez-Valero, and C. López-Martínez, "Serum and urine selenium concentrations as indicators of body status in patients with diabetes mellitus," Science of The Total Environment, vol. 228, no. 1, pp. 79-85, 1999.

[13] A. Simić, A. F. Hansen, B. O. Åsvold et al., "Trace element status in patients with type 2 diabetes in Norway: the HUNT3 Survey," Journal of Trace Elements in Medicine and Biology, vol. 41, pp. 91-98, 2017.

[14] M. Laclaustra, A. Navas-Acien, S. Stranges, J. M. Ordovas, and E. Guallar, "Serum selenium concentrations and diabetes in U.S. adults: national health and nutrition examination survey (NHANES) 2003-2004," Environmental Health Perspectives, vol. 117, no. 9, pp. 1409-1413, 2009.

[15] Z. Yuan, X. Xu, H. Ye, L. Jin, X. Zhang, and Y. Zhu, "High levels of plasma selenium are associated with metabolic syndrome and elevated fasting plasma glucose in a Chinese population: a case-control study," Journal of Trace Elements in Medicine and Biology, vol. 32, pp. 189-194, 2015.

[16] J. Bleys, A. Navas-Acien, and E. Guallar, "Serum selenium and diabetes in U.S. adults," Diabetes Care, vol. 30, no. 4, pp. 829-834, 2007.

[17] S. Stranges, S. Sieri, M. Vinceti et al., "A prospective study of dietary selenium intake and risk of type 2 diabetes," $B M C$ Public Health, vol. 10, no. 1, p. 564, 2010.

[18] J. Wei, C. Zeng, Q.-y. Gong et al., "The association between dietary selenium intake and diabetes: a cross-sectional study among middle-aged and older adults," Nutrition Journal, vol. 14, no. 1, p. 18, 2015.

[19] J. Zhou, K. Huang, and X. G. Lei, "Selenium and diabetesevidence from animal studies," Free Radical Biology and Medicine, vol. 65, pp. 1548-1556, 2013.
[20] Z. Li, X. Li, W. Ju et al., "High serum selenium levels are associated with impaired fasting glucose and elevated fasting serum glucose in Linyi, China," Journal of Trace Elements in Medicine and Biology, vol. 45, pp. 64-69, 2018.

[21] M. P. Rayman, "Food-chain selenium and human health: emphasis on intake," British Journal of Nutrition, vol. 100, no. 2, pp. 254-268, 2008.

[22] Institute of Medicine (US) Panel on Dietary Antioxidants and Related Compounds, Dietary Reference Intakes for Vitamin C, Vitamin E, Selenium, and Carotenoids, National Academies Press, Washington, DC, USA, 2000, https://www.ncbi.nlm. nih.gov/books/NBK225483/.

[23] M. Laclaustra, S. Stranges, A. Navas-Acien, J. M. Ordovas, and E. Guallar, "Serum selenium and serum lipids in US adults: national health and nutrition examination survey (NHANES) 2003-2004," Atherosclerosis, vol. 210, no. 2, pp. 643-648, 2010.

[24] L. Na, X. Wu, R. Feng et al., "The Harbin cohort study on diet, nutrition and chronic non-communicable diseases: study design and baseline characteristics," PLoS One, vol. 10, no. 4, Article ID e0122598, 2015.

[25] W. G. Yang and Y. Pan, China Food Composition, Peking University Medical Press, Beijing, China, 2009.

[26] D. R. Matthews, J. P. Hosker, A. S. Rudenski, B. A. Naylor, D. F. Treacher, and R. C. Turner, "Homeostasis model assessment: insulin resistance and ?-cell function from fasting plasma glucose and insulin concentrations in man," Diabetologia, vol. 28, no. 7, pp. 412-419, 1985.

[27] WHO, "Diabetes mellitus: report of a WHO study group," World Health Organization Technical Report Series, vol. 727, pp. 1-113, WHO, Geneva, Switzerland, 1985.

[28] K. Park, E. B. Rimm, D. S. Siscovick et al., "Toenail selenium and incidence of type 2 diabetes in U.S. men and women," Diabetes Care, vol. 35, no. 7, pp. 1544-1551, 2012.

[29] C. Kornhauser, J. R. Garcia-Ramirez, K. Wrobel, E.-L. PérezLuque, M.-E. Garay-Sevilla, and K. Wrobel, "Serum selenium and glutathione peroxidase concentrations in type 2 diabetes mellitus patients," Primary Care Diabetes, vol. 2, no. 2, pp. 81-85, 2008.

[30] S. Lenzen, J. Drinkgern, and M. Tiedge, "Low antioxidant enzyme gene expression in pancreatic islets compared with various other mouse tissues," Free Radical Biology and Medicine, vol. 20, no. 3, pp. 463-466, 1996.

[31] K. Ashton, L. Hooper, L. J. Harvey, R. Hurst, A. Casgrain, and S. J. Fairweather-Tait, "Methods of assessment of selenium status in humans: a systematic review," The American Journal of Clinical Nutrition, vol. 89, no. 6, pp. 2025s-2039s, 2009.

[32] S. Suresh, B. R. Prasad, B. Thomas, and A. Ramesh, "A comparative evaluation of antioxidant enzymes and selenium in the serum of periodontitis patients with diabetes mellitus type 2," Contemporary Clinical Dentistry, vol. 4, no. 2, pp. 176-180, 2013.

[33] X. Wang, W. Zhang, H. Chen et al., "High selenium impairs hepatic insulin sensitivity through opposite regulation of ROS," Toxicology Letters, vol. 224, no. 1, pp. 16-23, 2014.

[34] S. M. Lippman, E. A. Klein, P. J. Goodman et al., "Effect of selenium and vitamin $\mathrm{E}$ on risk of prostate cancer and other cancers: the selenium and vitamin E cancer prevention trial (SELECT)," JAMA, vol. 301, no. 1, pp. 39-51, 2009.

[35] S. Stranges, J. R. Marshall, R. Natarajan et al., "Effects of longterm selenium supplementation on the incidence of type 2 diabetes," Annals of Internal Medicine, vol. 147, no. 4, pp. 217-223, 2007.

[36] M. P. Longnecker, D. O. Stram, P. R. Taylor et al., "Use of selenium concentration in whole blood, serum, toenails, or 
urine as a surrogate measure of selenium intake," Epidemiology, vol. 7, no. 4, pp. 384-390, 1996.

[37] K. Rees, L. Hartley, C. Day, N. Flowers, A. Clarke, and S. Stranges, "Selenium supplementation for the primary prevention of cardiovascular disease," Cochrane Database Systematic Reviews, vol. 2013, no. 1, Article ID Cd009671, 2013.

[38] K. Hughes, M. Choo, P. Kuperan, C.-N. Ong, and T.-C. Aw, "Cardiovascular risk factors in non-insulin-dependent diabetics compared to non-diabetic controls: a population-based survey among Asians in Singapore," Atherosclerosis, vol. 136, no. 1, pp. 25-31, 1998.

[39] M. P. Rayman, G. Blundell-Pound, R. Pastor-Barriuso, E. Guallar, H. Steinbrenner, and S. Stranges, "A randomized trial of selenium supplementation and risk of type-2 diabetes, as assessed by plasma adiponectin," PLoS One, vol. 7, no. 9, Article ID e45269, 2012.

[40] J. Arnaud, M. de Lorgeril, T. Akbaraly et al., "Gender differences in copper, zinc and selenium status in diabetic-free metabolic syndrome European population-the IMMIDIET study," Nutrition, Metabolism and Cardiovascular Diseases, vol. 22, no. 6, pp. 517-524, 2012.

[41] O. Obeid, M. Elfakhani, S. Hlais et al., "Plasma copper, zinc, and selenium levels and correlates with metabolic syndrome components of Lebanese adults," Biological Trace Element Research, vol. 123, no. 1-3, pp. 58-65, 2008.

[42] S. Stranges, F. Galletti, E. Farinaro et al., "Associations of selenium status with cardiometabolic risk factors: an 8-year follow-up analysis of the Olivetti heart study," Atherosclerosis, vol. 217 , no. 1 , pp. 274-278, 2011.

[43] X. Chen, T. O. Scholl, M. J. Leskiw, M. R. Donaldson, and T. P. Stein, "Association of glutathione peroxidase activity with insulin resistance and dietary fat intake during normal pregnancy," The Journal of Clinical Endocrinology \& Metabolism, vol. 88, no. 12, pp. 5963-5968, 2003.

[44] H.-N. Kim and S.-W. Song, "Concentrations of chromium, selenium, and copper in the hair of viscerally obese adults are associated with insulin resistance," Biological Trace Element Research, vol. 158, no. 2, pp. 152-157, 2014.

[45] M. Ozkaya, M. Sahin, E. Cakal, K. Gisi, F. Bilge, and M. Kilinc, "Selenium levels in first-degree relatives of diabetic patients," Biological Trace Element Research, vol. 128, no. 2, pp. 144-151, 2009.

[46] S. F. A. Azab, S. H. Saleh, W. F. Elsaeed, M. A. Elshafie, L. M. Sherief, and A. M. H. Esh, "Serum trace elements in obese Egyptian children: a case-control study," Italian Journal of Pediatrics, vol. 40, no. 1, p. 20, 2014.

[47] I. Rotter, D. Kosik-Bogacka, B. Dołęgowska, K. Safranow, A. Lubkowska, and M. Laszczyńska, "Relationship between the concentrations of heavy metals and bioelements in aging men with metabolic syndrome," International Journal of Environmental Research and Public Health, vol. 12, no. 4, pp. 3944-3961, 2015.

[48] H. Gao, S. Hägg, P. Sjögren, P. C. Lambert, E. Ingelsson, and R. M. van Dam, "Serum selenium in relation to measures of glucose metabolism and incidence of type 2 diabetes in an older Swedish population," Diabetic Medicine, vol. 31, no. 7, pp. 787-793, 2014.

[49] P. Whanger, S. Vendeland, Y. C. Park, and Y. Xia, "Metabolism of subtoxic levels of selenium in animals and humans," Annals of Clinical and Laboratory Science, vol. 26, no. 2, pp. 99-113, 1996.
[50] M. Vinceti, E. T. Wei, C. Malagoli, M. Bergomi, and G. Vivoli, "Adverse health effects of selenium in humans," Reviews on Environmental Health, vol. 16, no. 4, pp. 233-251, 2001.

[51] C. Fürnsinn, R. Englisch, K. Ebner, P. Nowotny, C. Vogl, and W. Waldhäusl, "Insulin-like vs. non-insulin-like stimulation of glucose metabolism by vanadium, tungsten, and selenium compounds in rat muscle," Life Sciences, vol. 59, no. 23, pp. 1989-2000, 1996.

[52] J. P. McClung, C. A. Roneker, W. Mu et al., "Development of insulin resistance and obesity in mice overexpressing cellular glutathione peroxidase," Proceedings of the National Academy of Sciences, vol. 101, no. 24, pp. 8852-8857, 2004.

[53] X. Li, H. Chen, and P. N. Epstein, "Metallothionein and catalase sensitize to diabetes in nonobese diabetic mice: reactive oxygen species may have a protective role in pancreatic -cells," Diabetes, vol. 55, no. 6, pp. 1592-1604, 2006.

[54] X. D. Wang, M. Z. Vatamaniuk, S. K. Wang, C. A. Roneker, R. A. Simmons, and X. G. Lei, "Molecular mechanisms for hyperinsulinaemia induced by overproduction of seleniumdependent glutathione peroxidase-1 in mice," Diabetologia, vol. 51, no. 8, pp. 1515-1524, 2008.

[55] S. Satyanarayana, J. R. Sekhar, K. E. Kumar, L. B. Shannika, B. Rajanna, and S. Rajanna, "Influence of selenium (antioxidant) on gliclazide induced hypoglycaemia/anti hyperglycaemia in normal/alloxan-induced diabetic rats," Molecular and Cellular Biochemistry, vol. 283, no. 1-2, pp. 123-127, 2006.

[56] R. F. Burk, B. K. Norsworthy, K. E. Hill, A. K. Motley, and D. W. Byrne, "Effects of chemical form of selenium on plasma biomarkers in a high-dose human supplementation trial," Cancer Epidemiology Biomarkers \& Prevention, vol. 15, no. 4, pp. 804-810, 2006.

[57] H. Misu, T. Takamura, H. Takayama et al., "A liver-derived secretory protein, selenoprotein P, causes insulin resistance," Cell Metabolism, vol. 12, no. 5, pp. 483-495, 2010.

[58] Y. Mita, K. Nakayama, S. Inari et al., "Selenoprotein P-neutralizing antibodies improve insulin secretion and glucose sensitivity in type 2 diabetes mouse models," Nature Communications, vol. 8, no. 1, p. 1658, 2017.

[59] V. M. Labunskyy, B. C. Lee, D. E. Handy, J. Loscalzo, D. L. Hatfield, and V. N. Gladyshev, "Both maximal expression of selenoproteins and selenoprotein deficiency can promote development of type 2 diabetes-like phenotype in mice," Antioxidants \& Redox Signaling, vol. 14, no. 12, pp. 2327-2336, 2011.

[60] Y. Gao, H. C. Feng, and K. Walder, "Regulation of the selenoprotein SelS by glucose deprivation and endoplasmic reticulum stress-SelS is a novel glucose-regulated protein," FEBS Letters, vol. 563, no. 1-3, pp. 185-190, 2004.

[61] T. Manatkitsamran, P. Chattranukulchai, R. Chunhabundit et al., "Increased serum selenium levels and metabolic factors are associated with alterations in biomarkers of glucose metabolism," Journal of Nutrition Association of Thailand, vol. 54, no. 1, pp. 69-84, 2019.

[62] X. L. Wang, T.-b. Yang, J. Wei, G.-h. Lei, and C. Zeng, "Association between serum selenium level and type 2 diabetes mellitus: a non-linear dose-response meta-analysis of observational studies," Nutrition Journal, vol. 15, no. 1, p. 48, 2016.

[63] M. P. Rayman and S. Stranges, "Epidemiology of selenium and type 2 diabetes: can we make sense of it?," Free Radical Biology and Medicine, vol. 65, pp. 1557-1564, 2013. 
[64] Y. Zhang, H. Li, T. Lin et al., "Plasma selenium levels and risk of new-onset diabetes in hypertensive adults," Journal of Trace Elements in Medicine and Biology, vol. 56, pp. 6-12, 2019.

[65] D. Yu, D. Liang, L. Lei, R. Zhang, X. Sun, and Z. Lin, "Selenium geochemical distribution in the environment and predicted human daily dietary intake in northeastern Qinghai, China," Environmental Science and Pollution Research, vol. 22, no. 15, pp. 11224-11235, 2015.

[66] B. Lloyd, R. S. Lloyd, and B. E. Clayton, "Effect of smoking, alcohol, and other factors on the selenium status of a healthy population," Journal of Epidemiology \& Community Health, vol. 37, no. 3, pp. 213-217, 1983.

[67] F. Jossa, M. Trevisan, V. Krogh et al., "Serum selenium and coronary heart disease risk factors in southern Italian men," Atherosclerosis, vol. 87, no. 2-3, pp. 129-134, 1991. 


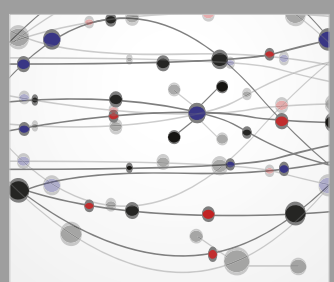

The Scientific World Journal
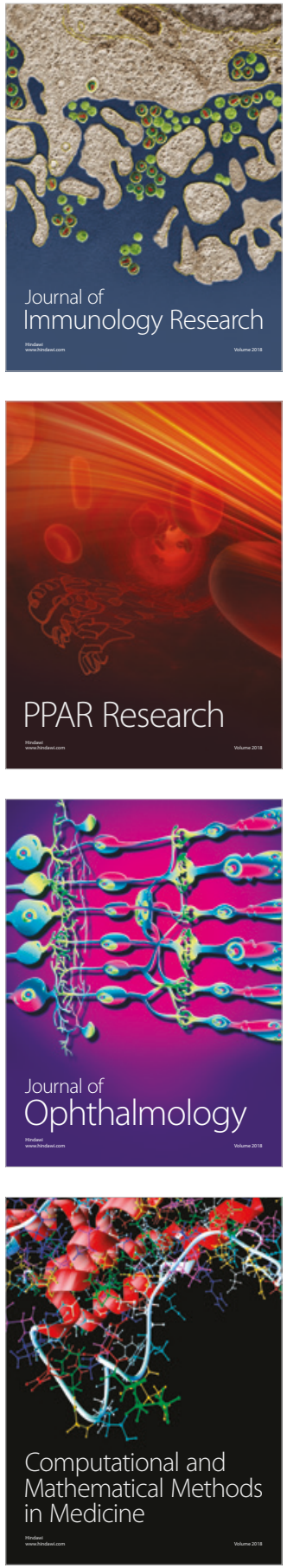

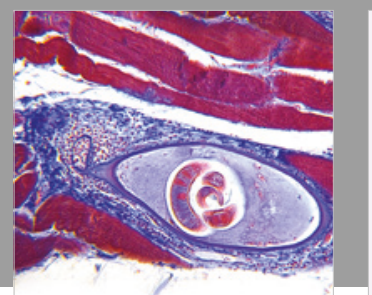

Gastroenterology Research and Practice

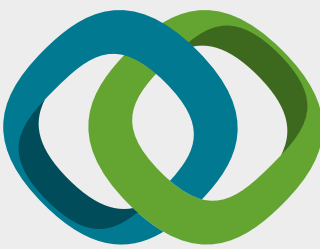

\section{Hindawi}

Submit your manuscripts at

www.hindawi.com
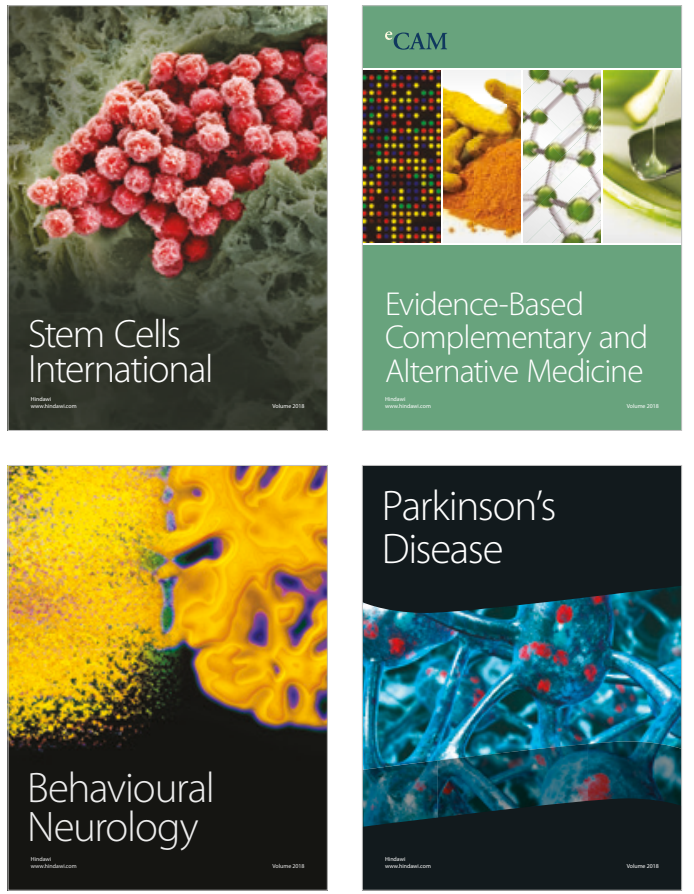

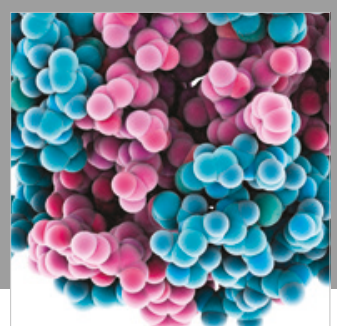

ournal of

Diabetes Research

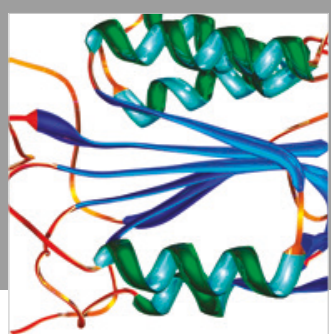

Disease Markers
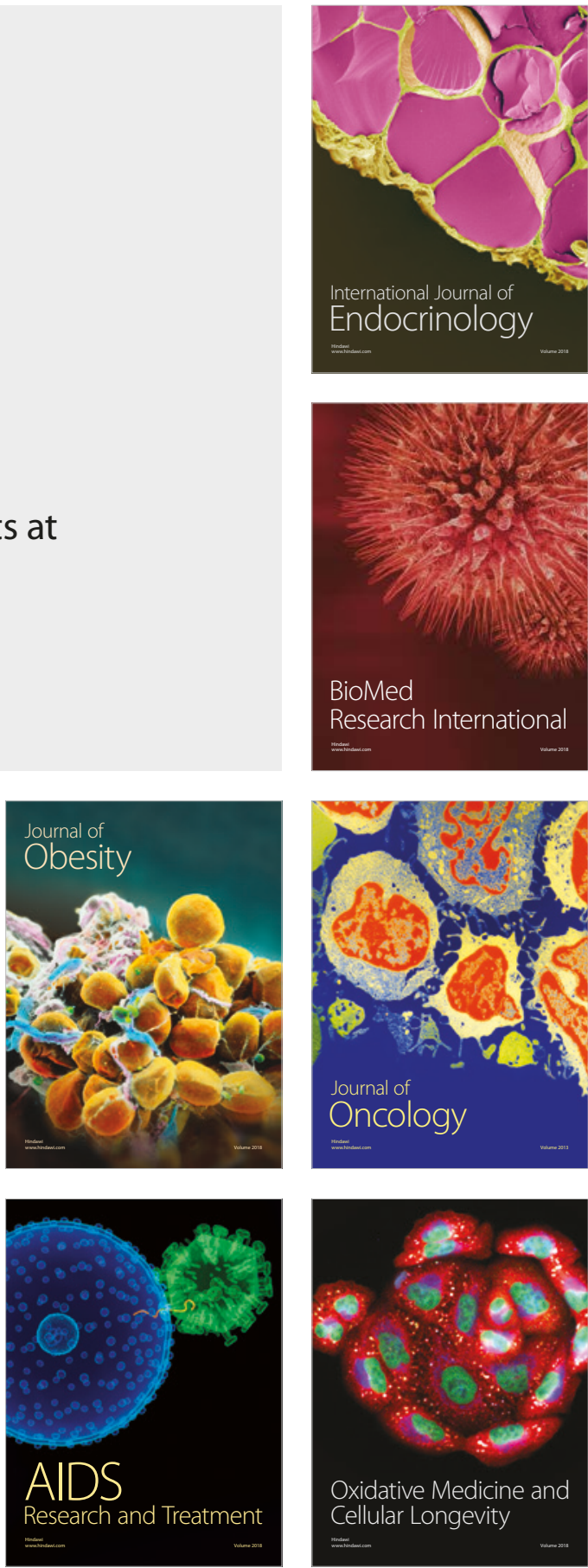